EPJ Web of Conferences 47, 15003 (2013)

DOI: $10.1051 /$ epjconf/20134715003

(C) Owned by the authors, published by EDP Sciences, 2013

\title{
Ultracool dwarf legacy science with ESA's Euclid mission
}

\author{
E.L. Martin ${ }^{1,2, a}$ \\ ${ }^{1}$ INTA-CSIC Centro de Astrobiologia, Torrejon de Ardoz, 28550 Madrid, Spain \\ ${ }^{2}$ ESA Independent Legacy Scientist for the Euclid Mission
}

\begin{abstract}
Euclid is an medium-class ESA mission that will carry out a 5 year survey of the extragalactic sky. The science drivers for the survey are cosmological and extragalactic. Nevertheless, the unprecedented depth over such a large area and the diffraction limited spatial resolution of the Euclid survey will have a long lasting impact in many fields of Astrophysics. This paper outlines the legacy science that will come out from Euclid in the field of ultracool dwarfs. In particular, it is foreseen that Euclid will increase the number of resolved ultracool binaries by more than an order of magnitude, and hence it will provide a stringent constraint on models of formation of very low-mass objects. Euclid can also find significant number of rare objects such as young free-floating planets or population III ultracool dwarfs.
\end{abstract}

\section{INTRODUCTION}

Brown dwarfs (BDs) represent a natural bridge between stars and giant planets, and hence they offer a natural testbed to study properties that are intermediate between those two types of objects. In the benchmark Pleiades cluster, the boundary between very low-mass (VLM) stars and BDs has been found to be located at spectral class dM6.5 according to the lithium test ([15], [28]). At dM7 spectral class there is a change in the strength of the molecular bands of $\mathrm{TiO}$ that dominate the optical spectrum of $\mathrm{M}$ dwarfs which is interpreted as evidence for the condensation of dust grains ([7]). Generically speaking all dwarfs with spectral types M7 and later are called ultra-cool dwarfs (UCDs), and they include both VLM stars and BDs.

In the solar vicinity, the last two decades have brought about a tremendous progress in the discovery and understanding of UCDs. Over 700 of these objects have been found all over the sky, and two new spectral classes, L and T, have been added to the century old MK system to classify objects cooler than M-type ([10, 16, 17]). These VLM objects are important for determining a complete census of the solar vicinity and to study the extension of the stellar mass function to very low-masses. There are strong indications in very young open clusters, such as Sigma Orionis, that the domain of BDs may extend down to just a few Jupiter masses [30], and that a numerous population of free-floating planets may be lurking in the Galaxy ([20, 29]).

UCD binaries have been resolved from space using the Hubble Space Telescope (HST; [18]; [3]), as well as from the ground using adaptive optics assisted imaging ([19]; [26]). These observations have indicated that the peak of the semimajor axis distribution of UCD binaries is located at smaller separations than for stellar binaries. The distribution of binary semimajor axis, mass ratios and eccentricity as a function of age, mass and metallicity is important to constrain models of star formation and evolution (e.g., [9]). In particular, one of the hot open issues is whether or not the properties of

\footnotetext{
${ }^{a}$ e-mail: ege@cab.inta-csic.es
}

This is an Open Access article distributed under the terms of the Creative Commons Attribution License 2.0, which permits unrestricted use, distribution, and reproduction in any medium, provided the original work is properly cited. 


\section{EPJ Web of Conferences}

VLM binaries are just a smooth continuation of the trends seen in stellar binaries or not. If there is a discontinuity in the binary properties it may be an indication that BDs form in a way different than stars.

The rest of this paper is organized as follows: In Sect. 2, we briefly describe the Euclid main survey and the ultracool dwarf legacy science that it will render possible. In Sect. 3, we mention other surveys that could be done with Euclid, in particular a transit survey of nearby ultracool dwarfs. In Sect. 4, we discuss the work that is needed to prepare for Euclid. In Sect. 5, we present our conclusions and acknowledgements.

\section{THE MAIN EUCLID SURVEY}

The Euclid mission is required to reach photometric limits in one optical and 3 near-infrared passbands of 24.5 magnitude (10 sigma) over an unprecedented wide area of the sky ( $\geq 15,000$ square degrees) and with diffraction limited image quality (FWHM $\leq 0.18$ arcsec at red optical wavelengths). Another important component of the Euclid main survey will be the slitless low-resolution $(\mathrm{R}=250)$ nearinfrared spectra.

Euclid is uniquely suited to discover new ultracool dwarfs (UCDs) by greatly extending the volume over which late-M, L, and T dwarfs can be detected. Our team has demonstrated that using Virtual Observatory tools with multiwalength survey data can be efficient to reveal reliable new UCDs ([1]) without the need for follow-up spectroscopy. Our team also has experience with using the maximum reduced proper motion method to select UCDs from large area surveys with high success rate (e.g., [27]), and with low-resolution spectroscopic characterization of UCDs and ultracool subdwarfs (e.g., [12]).

Ultracool subdwarfs represent the metal-poor analogues of solar-metallicity UCDs. They were discovered about a decade ago thanks to large-scale surveys like 2MASS, DENIS, and Sloan. They belong to the thick disk or halo and have large proper motions. They are very old and represent useful tracers of the Galactic chemical history. Their spectral energy distributions are as complex as their solarmetallicity counterparts with strong absorption bands, presence of neutral alkali but blue near-infrared colours due to the collision-induced $\mathrm{H} 2$ absorption. Only 9 sub-dwarfs have been discovered with L and $\mathrm{T}$ classes at the time of writing, showing that this area of substellar research is very recent and is likely to remain productive at the time of the advent of Euclid.

The VISTA Hemisphere Survey (VHS) will cover the entire Southern Hemisphere in J and K down to $\mathrm{J}($ Vega $)=19.5 \mathrm{mag}$. Current knowledge of UCDs and Monte-Carlo simulations suggest that VHS may uncover a several hundreds of new UCDs, implying that Euclid would increase that number by a factor of 500 for a depth of $\mathrm{J}=24 \mathrm{mag}$. Although less frequent that solar-metallicity dwarfs (about a factor of 5000, [14]) but easily distinguishable thanks to their high proper motion, hundreds of UCDs would be discovered by Euclid giving good enough statistics to (1) spot any difference(s) between the halo and solar-neighbourhood mass function, (2) discover very metal-poor UCDs, (3) investigate their spectral energy distributions, (4) constrain state-of-the-art atmospheric models at cool temperatures and a variety of metallicities, and (5) resolve binaries and determine the distribution of semimajor-axis and mass ratios as a function of age, mass and metallicity. More speculative, but not entirely out of question, is the detection of Population III UCDs which may have preserved a pristine chemical composition with nearly zero metallicity because they are less prone to accretion than their higher-mass counterparts ([5]).

Using the properties of UCD binaries from our HST surveys ([21]; [4]) and the expected spatial resolution of the Euclid main survey at red optical wavelengths, we have estimated that there will be about 3000 UCD binaries resolved with Euclid. Such an estimate is very preliminary because a more careful analysis of the Euclid performace needs to be done once the mission is better defined. Nevertheless, it is over an order of magnitude larger than the number of UCD binaries currently known, and it suggests that Euclid can provide the strongest statistical constrain on the binary properties as a function of age, mass and metallicity if those parameters are sufficiently well calibrated. 


\section{Hot Planets and Cool Stars}

\section{OTHER EUCLID SURVEYS}

A search for planetary transits in well-known nearby UCDs can be carried out with Euclid with a sensitivity down to very low-masses, even down to planet sizes smaller than the Moon. Continuous monitoring of carefully selected nearby UCDs during periods of several weeks would be useful to constrain the presence of habitable rocky planets around them. Current efforts using the NASA Kepler mission demostrate that the proposed investigation is feasible and suitable for using the Euclid observatory ([23]). In fact the near-infrared channel of Euclid has the advantage that cool dwarfs are observed to be more stable at near-infrared wavlengths than in the optical ([22], [6]). Transiting planets around nearby UCDs provide the best prospect for follow-up transmission spectroscopy, a very promising technique to characterize the atmospheric constituents of exoplanets ([8], [24]).

Our team has worked on the VLM population of several star-forming regions and young open clusters such as Lambda and Sigma Orionis, Pleiades, alpha Per, Taurus, IC348, NGC1333 and Upper Sco (e.g., [13]). Euclid images of open clusters are probably difficult to get because of issues with detection saturation and light scatter from bright stars. However, the dark nearby star-forming regions should be good targets for Euclid, and these data would be very valuable to compliment existing data and to extend the study of the substellar mass spectrum down to very low planetary masses. Ground-based optical/near-infrared images of those clusters and regions only have a sufficient sensitivity to detect VLM objects down to $\mathrm{J}=20$ th mag. For example, using ESO/VISTA data members of our team are reaching detection of objects down to 5 Jupiter masses in the Sigma Ori cluster ([2], [25]). In IC348 similar detection limits have been reached with CFHT/WIRCAM. Euclid imaging of those regions would provide a giant step forward in the detection of the very young planetary mass population, freefloating or at wide distances from stars.

\section{SCIENTIFIC PREPARATION OF EUCLID}

A spectroscopic library of benchmark UCDs in the Euclid fields is planned. It will cover a wide range of spectral types, surface gravities and metallicities. It will use the same instrumentation setup for all objects, and the same observing procedures and calibrations. Correction for telluric line absorption will be implemented so that the results can be used as reference for Euclid. The selected VLM objects will be faint to avoid any non-linearity or saturation effects with Euclid. At optical wavelengths the optimal instrumental combination available to us is GTC/OSIRIS. At near-infrared wavelengths it will be GTC/CIRCE or GTC/EMIR.

Team member JS is leading a high-precison astrometric monitoring program of faint L dwarfs with the ESO VLT and the GTC. The typical single epoch astrometric precision reached with the VLT is 0.1 milli-arcsec in the I-band. This program is an extension of the observations that ruled out the presence of a planet in the nearby VLM dwarf VB10 ([11]). Observations of faint UCDs in the Euclid fields are planned to provide a grid of astrometric reference objects. Observations of known UCD binaries with HST are also planned to calibrate the Euclid point spread function.

\section{CONCLUSIONS}

ESA's Euclid mission is poised to bring a long lasting legacy advance in the research of UCDs. Our team has been selected by ESA to prepare for Euclid, to mine the extragalactic surveys to find new UCDs, characterize them and to derive their binary properties. In addition our team is studying additional surveys that would exploit Euclid capabilities to investigate key aspects of UCD research. 
This work has been supported by the Spanish Ministerio de Economia y Competitivad through grant AyA201130147-C03-03. EM is thankful to the Geosciences department of the University of Florida for a visiting position during part of the time during which this manuscript was written. The members of the ESA independent legacy team on ultracool dwarfs are the following: David Barrado, Hervé Bouy, Nuria Huélamo, Nicolás Lodieu, Basmah Riaz, Johannes Sahlmann and Enrique Solano.

\section{References}

[1] Aberasturi M., Solano E. \& Martín E. L. Astronomy \& Astrophysics, 534, L7 (2011)

[2] Béjar V. J.S. et al., The Astrophysical Journal, 743, 21 (2011)

[3] Bouy H. et al., The Astronomical Journal, 126, 1526 (2003)

[4] Bouy H. et al., The Astrophysical Journal, 637, 1056 (2006)

[5] Johnson J.L. \& Khochfar S. Monthly Notices of the Royal Astronomical Society, 413, 1184 (2011)

[6] Goulding N.T. et al., Monthly Notices of the Royal Astronomical Society, 427, 358 (2012)

[7] Jones H. \& Tsuji T. The Astrophysical Journal, 480, L39 (1997)

[8] Kaltenegger L. \& Traub W. A. The Astrophysical Journal, 698, 519 (2009)

[9] King R.R. et al., Monthly Notices of the Royal Astronomical Society, 427, 2636 (2012)

[10] Kirkpatrick J. D. et al., The Astrophysical Journal, 519, 802 (1999)

[11] Lazorenko P.F. et al., Astronomy \& Astrophysics, 527, A25 (2011)

[12] Lodieu N. et al., The Astrophysical Journal, 708, L107 (2010)

[13] Lodieu N. et al., Monthly Notices of the Royal Astronomical Society, 418, 2604 (2011)

[14] Lodieu N. et al., Astronomy \& Astrophysics, 542, 105 (2012)

[15] Martín E. L. et al., The Astrophysical Journal, 469, 706 (1996)

[16] Martín E. L. et al., Astronomy \& Astrophysics, 327, L29 (1997)

[17] Martín E. L. et al., The Astronomical Journal, 118, 2466 (1999)

[18] Martín E. L., Brandner W., \& Basri G. Science, 283, 1718 (1999)

[19] Martín E. L. et al., The Astrophysical Journal, 529, L37 (2000)

[20] Martín E. L. et al., The Astrophysical Journal, 558, L117 (2001)

[21] Martín E. L. et al., Astronomy \& Astrophysics, 456, 253 (2006)

[22] Martín E. L. et al., The Astrophysical Journal, 644, L75 (2006)

[23] Martín E. L. et al., Astronomy \& Astrophysics, submitted (2013)

[24] Murgas F. et al., Astronomy \& Astrophysics, 544, A41 (2012)

[25] Peña Ramírez K. et al., The Astrophysical Journal, 754, 33 (2012)

[26] Potter D. et al., The Astrophysical Journal, 567, L133 (2002)

[27] Phan Bao N. et al., Monthly Notices of the Royal Astronomical Society, 383, 831 (2008)

[28] Rebolo R. et al., The Astrophysical Journal, 469, L53 (1996)

[29] Sumi T. et al., Nature, 473, 349 (2011)

[30] Zapatero Osorio M.R. et al., Science, 290, 103 (2000) 